\title{
DGTD for a Class of Low-Observable Targets: A Comparison with MoM and (2,2) FDTD
}

\author{
Jesus Alvarez, Student Member, IEEE, J. M. Alonso-Rodriguez, H. Carbajosa-Cobaleda, Miguel R. Cabello \\ Luis D. Angulo, R. Gomez Martin, Senior Member, IEEE, and Salvador G. Garcia, Member, IEEE
}

\begin{abstract}
The simulation of low-observable targets requires high accuracy, both in the geometrical discretization as well as in the numerical solution of the electromagnetic problem. In this letter, we employ the well-known NASA almond, to illustrate the accuracy of the Leap-Frog Discontinuous Galerkin method, combined with a local time stepping algorithm, comparing it with the MoM and the $(2,2)$ FDTD methods.
\end{abstract}

Index Terms- Discontinuous Galerkin, Time-domain analysis, RADAR cross-section.

\section{INTRODUCTION}

The analysis of the electromagnetic scattering by low observable (LO) targets is a challenging problem for numerical solvers. Frequency Domain (FD) methods, like the Method of Moments (MoM) [1], are a common choice to accurately deal with these problems. However, MoM-FD methods become computationally inefficient for wideband computations, since each frequency needs a complete simulation. Time-domain (TD) methods are an attractive alternative, since they employ a marching-on-in-time algorithm that permits to find the whole FD behavior with a single simulation. Among TD methods, the Finite-Difference Time-Domain (FDTD) method [2] has become very popular for its versatility and power, though its staircased nature imposes a significant constraint on the discretization of arbitrary curvatures and intricate details. Finite Element Methods in TD (FEMTD) [3] permit to overcome these limitations, thanks to the use of unstructured meshes to handle geometrical details. Nevertheless, they are computationally intensive because of their implicit nature, which requires the solution of a sparse linear system of equations at each step of the time marching procedure. Explicit FEMTD algorithms have been proposed based on sparse approximate inverses, efficiently implemented on parallel machines [4].

The Discontinuous Galerkin Time Domain (DGTD) methods are currently attracting an increasing attention [5], for combining some of the advantages of FDTD and FEMTD methods. The main difference between DGTD and other FEMTD methods, is that the solution is allowed to be discontinuous across the boundaries between adjacent elements,

The work described in this paper and the research leading to these results has received funding from the European Community's Seventh Framework Programme FP7/2007-2013, under grant agreement no 205294 (HIRF SE project), and from the Spanish National Projects TEC2010-20841-C04-04, CSD2008-00068, and the Junta de Andalucia Project P09-TIC-5327.

The first three authors are with Cassidian, EADS-CASA, Avda. John Lennon s/n. 28906 Getafe, Spain. The other authors are with the Dept. of Electromagnetism, University of Granada, Fuentenueva s/n, 18071 Granada, Spain, Corresponding author: e-mail: salva@ ugr.es, Ph.: +34 958 240507, fax: +34958 242353 . which communicate by means of numerical fluxes. The result are computationally affordable and accurate TD algorithms.

In this letter, we apply a DGTD method [6], [7] based on the Leap-Frog (LF) time integration scheme (LFDG) and combined with a Local Time Stepping (LTS) strategy, to calculate the RCS of PEC and coated NASA almonds. This geometry has been chosen for being a challenging example of LO target used in the validation of numerical solvers [8]. Results show that the LTS-LFDG method can be competitive with MoM-FD, and the (2,2) FDTD, methods, in terms of accuracy vs. computational time.

\section{LFDG FUNDAMENTALS}

Let us begin by describing briefly the fundamentals of the DGTD method (further details of the implementation used by the authors can be found in [6], [7], [9], [10]). The DGTD method is based on a finite-element geometrical discretization of the space into $M$ non-overlapping elements $V^{m}$, where we define element-by-element a basis of local continuous vector test functions $\left(\mathcal{B}^{m}=\left\{\boldsymbol{\Phi}_{1}^{m}, \boldsymbol{\Phi}_{2}^{m}, \ldots, \boldsymbol{\Phi}_{Q}^{m}\right\}\right)$, used both to expand the electromagnetic field, and as test functions to find a weak form of Maxwell curl equations (Galerkin procedure). For lossless linear isotropic homogeneous media, we have

$$
\begin{gathered}
\left\langle\boldsymbol{\Phi}_{q^{\prime}}^{m}, \mu \partial_{t} \mathbf{H}^{m}+\nabla \times \mathbf{E}^{m}+\sigma_{m} \mathbf{H}\right\rangle_{V^{m}}=0 \\
\left\langle\boldsymbol{\Phi}_{q^{\prime}}^{m}, \varepsilon \partial_{t} \mathbf{E}^{m}-\nabla \times \mathbf{H}^{m}+\sigma_{e} \mathbf{E}\right\rangle_{V^{m}}=0 \\
\forall q=(1, \ldots, Q), \quad m=(1, \ldots, M)
\end{gathered}
$$

with $\mathbf{E}, \mathbf{H}, \sigma_{e}, \sigma_{m}, \varepsilon, \mu$ being, respectively: the electric and magnetic field, the electric and magnetic conductivity, permittivity, and permeability. Applying the discontinuous Galerkin method [5] to Eqs. (1) and (2), we can formulate the following semi-discrete spatial algorithm:

$$
\begin{aligned}
& \mu \mathbb{M} d_{t} H^{m}+\left(\sigma_{m} \mathbb{M}-\mathbb{F}_{\nu h}\right) H^{m}+\mathbb{F}_{\nu h}^{+} H^{m+}=\left(\mathbb{F}_{\kappa e}-\mathbb{S}\right) E^{m}-\mathbb{F}_{\kappa e}^{+} E^{m+} \\
& \varepsilon \mathbb{M} d_{t} E^{m}+\left(\sigma_{e} \mathbb{M}-\mathbb{F}_{\nu e}\right) E^{m}+\mathbb{F}_{\nu e}^{+} E^{m+}=\left(\mathbb{S}-\mathbb{F}_{\kappa h}\right) H^{m}+\mathbb{F}_{\kappa h}^{+} H^{m+}
\end{aligned}
$$

where $H^{m}$ and $E^{m}$ are column vectors with the degrees of freedom (dofs) at the element $m$, and $H^{m+}$ and $E^{m+}$ the dofs at the adjacent elements. $\mathbb{M}$ is the mass matrix, $\mathbb{S}$ is the stiffness matrix, and $\mathbb{F}$ are the flux matrices [11]. The resulting method has a spatial error behaving as $O\left(h^{2 p+1}\right)$, with $h$ a measure of the size of the elements, and $p$ the order of the basis functions [6].

The time integration, can be performed in different manners [5]. In this paper, we use a $2^{\text {nd }}$-order Leap-Frog (LF) scheme, 
which employs a centered approximation for the time derivatives $\left(d_{t} U_{n}^{m} \approx \Delta t^{-1}\left(U_{n+\frac{1}{2}}^{m}-U_{n-\frac{1}{2}}^{m}\right)\right)$ in (3), to yield ${ }^{1}$

$$
\begin{aligned}
H_{n+\frac{1}{2}}^{m}= & \alpha_{m} H_{n-\frac{1}{2}}^{m}+\beta_{m} \mathbb{M}^{-1}\left[-\left(\mathbb{S}-\mathbb{F}_{\kappa e}\right) E_{n}^{m}-\mathbb{F}_{\kappa e}^{+} E_{n}^{m+}+\right. \\
& \left.\mathbb{F}_{\nu h} H_{n-\frac{1}{2}}^{m}-\mathbb{F}_{\nu h}^{+} H_{n-\frac{1}{2}}^{m+}-M_{n}^{s \kappa}+J_{n}^{s \nu}\right] \\
E_{n+1}^{m}= & \alpha_{e} E_{n}^{m}+\beta_{e} \mathbb{M}^{-1}\left[\left(\mathbb{S}-\mathbb{F}_{\kappa h}\right) H_{n+\frac{1}{2}}^{m}+\mathbb{F}_{\kappa h}^{+} H_{n+\frac{1}{2}}^{m+}+\right. \\
& \left.\mathbb{F}_{\nu e} E_{n}^{m}-\mathbb{F}_{\nu e}^{+} E_{n}^{m+}-J_{n+\frac{1}{2}}^{s \kappa}-M_{n+\frac{1}{2}}^{s \nu}\right]
\end{aligned}
$$

where the expressions for the constants are

$$
\begin{aligned}
\alpha_{m}=\frac{1-\frac{\Delta t \sigma_{m}}{2 \mu}}{1+\frac{\Delta t \sigma_{m}}{2 \mu}}, & \beta_{m}=\frac{\Delta t}{\mu\left(1+\frac{\Delta t \sigma_{m}}{2 \mu}\right)} \\
\alpha_{e}=\frac{1-\frac{\Delta t \sigma_{e}}{2 \varepsilon}}{1+\frac{\Delta t \sigma_{e}}{2 \varepsilon}}, & \beta_{e}=\frac{\Delta t}{\varepsilon\left(1+\frac{\Delta t \sigma_{e}}{2 \varepsilon}\right)}
\end{aligned}
$$

Local time-stepping strategies have been efficiently incorporated into the LF stepping procedure [12], [13] to alleviate the computational overload driven by the conditional stability of LF in real problems. Here, we use the LTS algorithm described in [9], [10], to arrange the mesh elements in different tiers, according to the maximum time step allowed for stability, so that different time steps can be used for each tier. An interpolation procedure is used at the interface between tiers.

\section{MOM CCIE FUNDAMENTALS}

The MoM used in this comparison is applied to the Current and Change Integral Equation (CCIE) [14], combined with a Multilevel Fast Multipole Method (MLFMM) [15] to efficiently perform the matrix-vector products. CCIE introduces electric and magnetic surface charges densities, apart from the surface current densities of the Poggio-Miller-ChanHarrington-Wu-Tsai (PM-CHWT) method [16], and solves a system of four integral equations for all four unknowns. The resulting scheme is well conditioned and leads to fast convergences with iterative solvers on a wide frequency range. Let us briefly summarize its fundamentals.

The time-harmonic $\left(e^{j \omega t}\right)$ total electric and magnetic fields can be expressed on the surface of a homogeneous body as a function of the electric and magnetic surface charges densities $(\mathbf{J}, \mathbf{M})$, and the electric and magnetic surface charges densities $\left(\rho_{e}, \rho_{m}\right)$ as,

$$
\begin{aligned}
& \mathbf{E}=\mathbf{E}^{i n}-j \omega \mu \mathcal{S}(\mathbf{J})+\frac{\mu}{j \omega \varepsilon} \mathcal{N}\left(\rho_{e}\right)-\mathcal{K}(\mathbf{M}) \\
& \mathbf{H}=\mathbf{H}^{i n}-j \omega \varepsilon \mathcal{S}(\mathbf{M})+\frac{\varepsilon}{j \omega \mu} \mathcal{N}\left(\rho_{m}\right)+\mathcal{K}(\mathbf{J})
\end{aligned}
$$

with $\mathbf{E}^{i n}$ and $\mathbf{H}^{i n}$ being the incident fields, $\hat{\mathbf{n}}$ the inner unit normal of the surface, and $\mathcal{S}, \mathcal{N}$ and $\mathcal{K}$ the surface integral

\footnotetext{
${ }^{1} \mathrm{~A}$ backward approximation for the terms $H_{n}^{m} \approx H_{n-\frac{1}{2}}^{m}$ and $E_{n+\frac{1}{2}}^{m} \approx$ $E_{n}^{m}$, and an average approximation for the conductive terms $H_{n}^{m^{2}} \approx$ $\frac{1}{2}\left(H_{n+\frac{1}{2}}^{m}+H_{n-\frac{1}{2}}^{m}\right)$ and $E_{n+\frac{1}{2}}^{m} \approx \frac{1}{2}\left(E_{n+1}^{m}+E_{n}^{m}\right)$ are also used.
}

operators,

$$
\begin{aligned}
\mathcal{S}(\mathbf{f})(\mathbf{r}) & =\int G\left(\mathbf{r}, \mathbf{r}^{\prime}\right) \mathbf{f}\left(\mathbf{r}^{\prime}\right) d s\left(\mathbf{r}^{\prime}\right) \\
\mathcal{N}(f)(\mathbf{r}) & =\int \nabla G\left(\mathbf{r}, \mathbf{r}^{\prime}\right) f\left(\mathbf{r}^{\prime}\right) d s\left(\mathbf{r}^{\prime}\right) \\
\mathcal{K}(\mathbf{f})(\mathbf{r}) & =\nabla \times \mathcal{S}(\mathbf{f})(\mathbf{r})
\end{aligned}
$$

$G\left(\mathbf{r}, \mathbf{r}^{\prime}\right)=\frac{e^{-j k R}}{4 \pi R}, R=\left|\mathbf{r}-\mathbf{r}^{\prime}\right|$, is the usual free-space Green function, with $k=\omega \sqrt{\varepsilon \mu}$.

Considering the usual boundary conditions at the interface between two media (1 and 2),

$$
\begin{aligned}
& \hat{\mathbf{n}}_{2} \cdot\left(\varepsilon_{2} \mathbf{E}_{\mathbf{2}}-\varepsilon_{1} \mathbf{E}_{\mathbf{1}}\right)=\rho_{e}, \hat{\mathbf{n}}_{2} \cdot\left(\mu_{2} \mathbf{H}_{\mathbf{2}}-\mu_{1} \mathbf{H}_{\mathbf{1}}\right)=\rho_{m} \\
& \hat{\mathbf{n}}_{2} \times\left(\mathbf{H}_{\mathbf{2}}-\mathbf{H}_{\mathbf{1}}\right)=\mathbf{J}, \hat{\mathbf{n}}_{2} \times\left(\mathbf{E}_{\mathbf{2}}-\mathbf{E}_{\mathbf{1}}\right)=-\mathbf{M}
\end{aligned}
$$

four surface Fredholm integral equations of the second kind can be formulated for the tangential and normal components of the fields,

$\left(\begin{array}{c}\varepsilon \mathbf{E}_{\mathbf{n}}^{\text {in }} \\ \mu \mathbf{H}_{\mathbf{n}}^{\text {in }} \\ \mathbf{H}_{\mathbf{t}}^{\text {in }} \\ -\mathbf{E}_{\mathbf{t}}^{\text {in }}\end{array}\right)=\left(\begin{array}{cccc}\mathcal{I}-\frac{\mu}{j \omega \varepsilon} \mathcal{N}_{n} & 0 & j \omega \mu \mathcal{S}_{n} & \mathcal{K}_{n} \\ 0 & \mathcal{I}-\frac{\varepsilon}{j \omega \mu} \mathcal{N}_{n} & -\mathcal{K}_{n} & j \omega \varepsilon \mathcal{S}_{n} \\ 0 & -\frac{\varepsilon}{j \omega \mu} \mathcal{N}_{t} & \mathcal{I}-\mathcal{K}_{t} & j \omega \varepsilon \mathcal{S}_{t} \\ \frac{\mu}{j \omega \varepsilon} \mathcal{N}_{t} & 0 & -j \omega \mu \mathcal{S}_{t} & \mathcal{I}-\mathcal{K}_{t}\end{array}\right)\left(\begin{array}{c}\rho_{e} \\ \rho_{m} \\ \mathbf{J} \\ \mathbf{M}\end{array}\right)$

where $\mathbf{F}_{n}=\hat{\mathbf{n}} \cdot \mathbf{F}$ and $\mathbf{F}_{t}=\hat{\mathbf{n}} \times \mathbf{F}$. This set of equations together with the continuity conditions

$$
\nabla \cdot \mathbf{J}+j w \rho_{e}=0, \nabla \cdot \mathbf{M}+j w \rho_{m}=0
$$

form the CCIE system, which can be numerically solved by making use of the MoM method. Similarly to the CFIE, which combines EFIE and MFIE, a combined form of the CCIE is formulated, resulting into the CCCIE described in [14]. The continuity equations are taken into account by directly adding a combination of the null (12) to $\rho_{e}$ and $\rho_{m}$ of (8). This combination is crucial for the accurate behavior of the scheme along the whole frequency range [14]. The final algorithm is found by expanding the scalar unknowns $\left(\rho_{e}, \rho_{m}\right)$ with pulse functions, and the vector unknowns $(\mathbf{J}, \mathbf{M})$ with the classical Rao-Wilton-Glisson (RWG) basis functions. In the same manner, the equations of rows 1 and 2 are tested with pulse functions, and rows 3 and 4 with RWG ones.

\section{NASA ALMOND BENCHMARK}

In this section, we find the Radar Cross-Section (RCS) of a typical LO target: the NASA almond. This geometry is a benchmark of the Electromagnetic Code Consortium, used for validation purposes [8]. The LTS-LFDG method [6], the MoM-MLFMM for CCCIE (HPTESP-MAT Cassidian tool, certified for RCS calculation by the Spanish Military Airworthiness Authority INTA [17]), and the well-known (2,2) FDTD method (UGRFDTD MPI/OpenMP parallel code [18], validated under the 7PM EU HIRF-SE project [19]), have been employed for this purpose. 


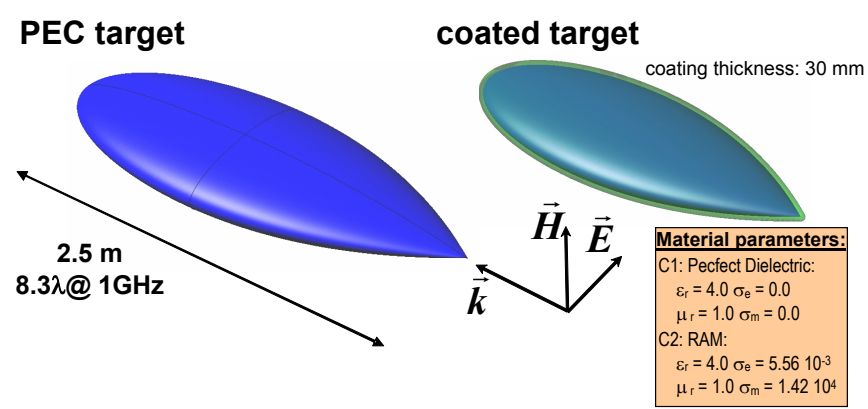

Fig. 1. Geometry of the NASA almond.

The NASA almond (Fig.1) is composed by

Half ellipsoid: $-0.416667<t<0.0$ and $-\pi<\psi<\pi$

$$
\left\{\begin{array}{l}
x=d t \\
y=0.193333 d\left(\sqrt{1-\left(\frac{t}{0.416667}\right)^{2}}\right) \cos \psi \\
z=0.06444 d\left(\sqrt{1-\left(\frac{t}{0.416667}\right)^{2}}\right) \sin \psi
\end{array}\right.
$$

Half elliptic ogive: $-0.0<t<0.583333$ and $-\pi<\psi<\pi$

$$
\left\{\begin{array}{l}
x=d t \\
y=4.833450 d\left(\sqrt{1-\left(\frac{t}{2.083350}\right)^{2}}-0.96\right) \cos \psi \\
z=1.611148 d\left(\sqrt{1-\left(\frac{t}{2.083350}\right)^{2}}-0.96\right) \sin \psi
\end{array}\right.
$$

where $d=2.5 \mathrm{~m}$, is the length of the structure. Note that this is a complete double curvature geometry, where we can find, both smoothly and sharply curved zones, as well as a singular point, the ogive vertex. Apart from a PEC case, two different coated material cases have been studied: with a perfect dielectric, and with a Radar Absorber Material (RAM), proposed under JINA 2006 [20] (see Fig. 1 for details). For the LTS-LFDG method, we have discretized the surface with curvilinear $2^{\text {nd }}$ order tetrahedrons. Care has been taken for the discretization close to the vertex by defining small elements (low value of h), as an a priori level of h-refinement (see Fig. 2). Apart from the vertex, we have defined a maximum element size $h$ during the mesh-generation process, corresponding to the value of $\frac{h}{\lambda}=0.4$ of the maximum frequency, which is efficient in terms of computational and required accuracy. Once we have generated the mesh, the order $p$ in each element is chosen depending on the element size, assigning the minimum $p$ that meets the required accuracy [10]. For instance, in the simplest case (PEC, bistatic RCS at $1 \mathrm{GHz}$ ), the mesh was composed of 2018928 elements: 785678 had $p=1,523786$ had $p=2$, and 709464 had $p=3$, being the total number of unknowns $18710^{6}$. We do not use orders $p$ higher than 3 since have been found not to be optimum in terms of computational cost and accuracy [10].

The simulation region is divided into a total-field zone, holding the almond, and a scattered-field zone. The surface between both regions serve to excite the plane-wave by Huygens sources, through the flux terms in a weak way [11]. The same

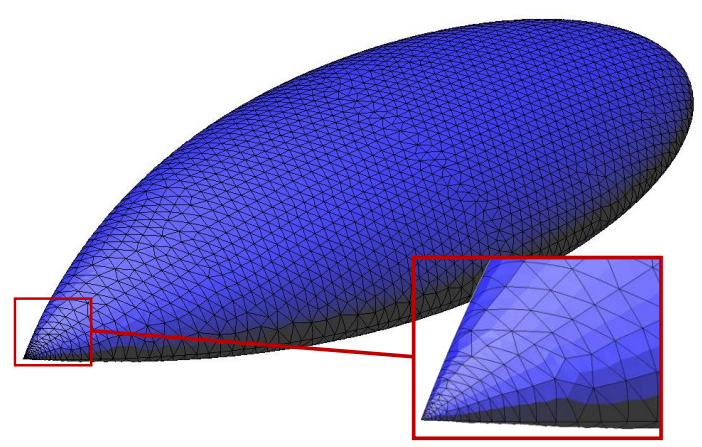

Fig. 2. Snapshot of the mesh used for the PEC case computation. Only the surface mesh is shown.

surface is used to compute the near-to-far-field transformation and to calculate the RCS. Conformal PMLs [7], [21] are used to truncate the whole domain.

The structures are illuminated with a horizontally-polarized plane wave, impinging on the almond at the vertex. The resulting copolar bistatic RCS at $1 \mathrm{GHz}$, computed with LTSLFDG and compared the results with MoM, are shown in Fig. 3 for the three cases analyzed, with excellent agreement. The monostatic RCS from $500 \mathrm{MHz}$ to $2 \mathrm{GHz}$ is shown in Fig. 5. Excellent agreement is again found both for PEC and C2 (RAM material) cases. Minor differences are detected for the $\mathrm{C} 1$ (perfect dielectric) case. It is important to note that this is a challenging case for MoM, where the required number of iterations to solve iteratively the MoM linear system is quite high, and the number of unknowns cannot be too high in order to have a solution with affordable computational costs. Notice that the whole frequency band computation requires 301 runs. The minor differences found so far are, in our opinion, due to the use of a coarse mesh in the MoM computations.

In Fig. 4, we have also compared the PEC case with uniform-mesh FDTD simulations with a $1.5 \mathrm{~mm}$ cell length. A brute-force solution has been obtained with $(2,2)$ FDTD just for comparison purposes (higher-order stencils, uneven meshing, subgridding or conformal techniques, combined with FDTD are not used here, though they are well-known to improve the results and reduce the computational costs). Both for FDTD and LTS-LFDG, we use a padding of half a wavelength at $1 \mathrm{GHz}$ between the almond and the PML region, and we simulate $50 \mathrm{nsec}$ of the transient response. The FDTD problem employs 750 MCells (6 $10^{9}$ unknowns) and requires a CPU time of 24 hours in a 12 core Intel Xeon X5520 $2.26 \mathrm{Ghz}$ architecture, while the LTS-LFDG code only requires 18 hours. No computer resources are shown for the HPTESPMAT, for industrial property rights protection. The reader is referred to [22] for typical figures of MoM methods. Results for the bistatic RCS at $1 \mathrm{GHz}$ confirm, as also stated by the authors in [11], the superior accuracy of LTS-LFDG especially near the LO (monostatic) zone.

\section{Conclunsions}

In this letter, we have shown the application of three numerical solvers, based on the LTS-LFDG, MoM CCCIE and FDTD methods, to the prediction of the RCS of a typical 


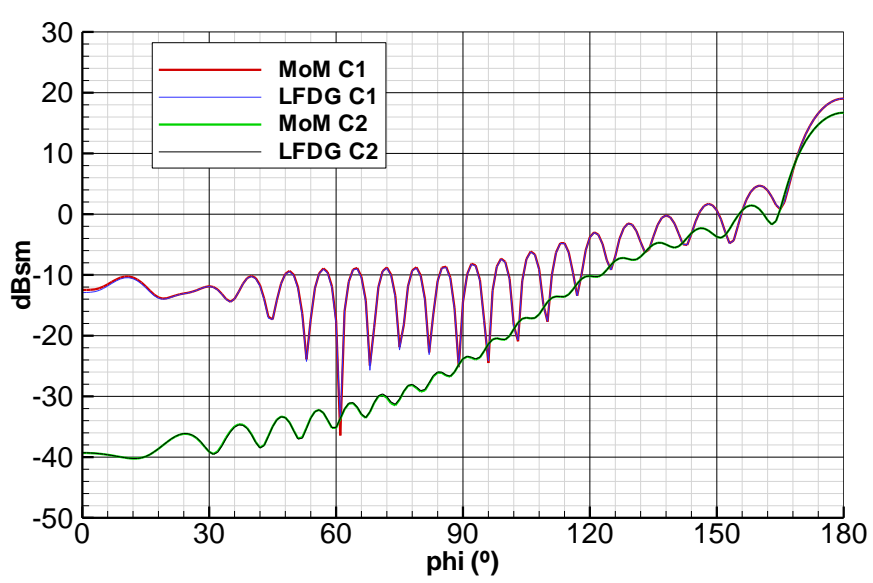

Fig. 3. Bistatic Radar Cross Sections of the NASA almond at $1 \mathrm{GHz}$. Comparison results between LTS-LFDG and MoM for the coated almond.

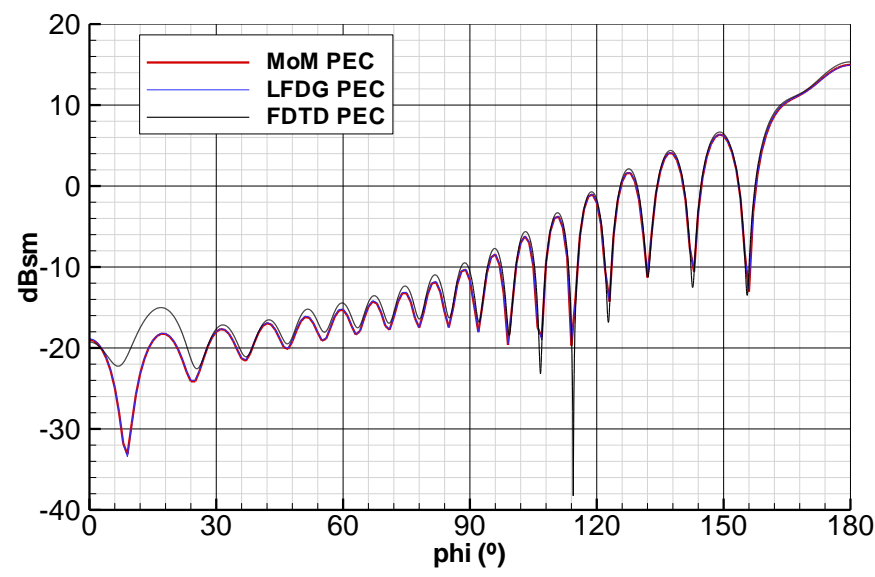

Fig. 4. Bistatic Radar Cross Sections of the NASA almond at $1 \mathrm{GHz}$. Comparison results between LTS-LFDG, MoM and FDTD for the PEC case.

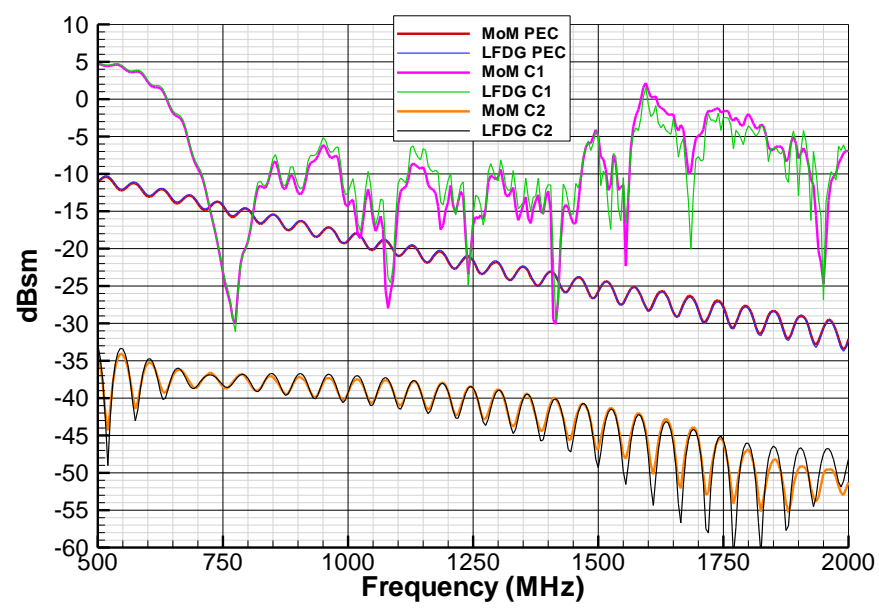

Fig. 5. Monostatic Radar Cross Sections of the NASA almond. Comparison results between LTS-LFDG (1 computation per case) and MoM (301 frequencies/computations per case).
LO target: the NASA almond. The accuracy of the LTSLFDG has been demonstrated to be in the range of that of the MoM CCIE method, outperforming the classical (uniformmesh, second-order) FDTD method in terms of computational time vs. accuracy.

\section{REFERENCES}

[1] R. F. Harrington, Field Computation by Moment Methods. New York: Macmillan, 1968

[2] K. S. Yee, "Numerical solution of initial boundary value problems involving Maxwell's equations in isotropic media," IEEE Transactions on Antennas and Propagation, vol. Ap14, no. 3, pp. 302-307, 1966.

[3] J. F. Lee, R. Lee, and A. Cangellaris, "Time-domain finite-element methods," IEEE Transactions on Antennas and Propagation, vol. 45, no. 3, pp. 430-442, March 1997.

[4] J. Kim and F. L. Teixeira, "Parallel and explicit finite element timedomain method for Maxwell's equations," IEEE Transactions on Antennas and Propagation, vol. 59, no. 6, pp. 2350-2356, 2011.

[5] J. S. Hesthaven and T. Warburton, Nodal Discontinuous Galerkin Methods. Algorithms, Analysis, and Applications. Springer Science+Business Media, LLC, 233 Spring Street, New York, NY 10013, USA: Springer, 2008.

[6] J. Alvarez, L. D. Angulo, A. R. Bretones, and S. G. Garcia, "A spuriousfree Discontinuous Galerkin Time-Domain method for the accurate modeling of microwave filters," IEEE Transactions on Microwave Theory and Techniques, vol. 60, no. 6, pp. 2359-2369, 2012.

[7] - "A leap-frog discontinuous Galerkin time-domain method for HIRF assessment," IEEE Transactions on Electromagnetic Compatibilbity, vol. (accepted), 2013.

[8] A. C. Woo, H. T. G. Wang, M. J. Schuh, and M. L. Sanders, "Benchmark radar targets for the validation of computational electromagnetic programs," IEEE Antennas and Propagation Magazine, vol. 35, no. 1, pp. 84-89, February 1993.

[9] J. Alvarez, L. D. Angulo, A. R. Bretones, C. M. de Jong, and S. G. Garcia, "An efficient local time?stepping DGTD method: application to antenna modeling," IEEE Transactions on Antennas and Propagation, (submitted).

[10] J. Alvarez, "A discontinuous galerkin finite element method for the timedomain solution of maxwell equations," Ph.D. dissertation, University of Granada (Spain), 2013.

[11] J. Alvarez, L. D. Angulo, M. F. Pantoja, A. R. Bretones, and S. G. Garcia, "Source and boundary implementation in vector and scalar DGTD," IEEE Transactions on Antennas and Propagation, vol. 58, no. 6, pp. 1997-2003, 2010.

[12] E. Montseny, S. Pernet, X. Ferriéres, and G. Cohen, "Dissipative terms and local time-stepping improvements in a spatial high order discontinuous Galerkin scheme for the time-domain Maxwell's equations," Journal of Computational Physics, vol. 227, pp. 6795-6820, 2008.

[13] S. Piperno, "DGTD methods using modal basis functions and symplectic local time-stepping: Application to wave propagation problems," Journal of Computational Physics, vol. 217, pp. 340-363, 2006.

[14] M. Taskinen and P. Yla-Oijala, "Current and charge integral equation formulation," IEEE Transactions on Antennas and Propagation, vol. 54, no. 1, pp. 58-67, January 2006.

[15] W. C. Chew, J. M. Jin, E. Michielssen, and J. M. Song, Fast and Efficient Algorithms in Computational Electromagnetics. Boston / London: Artech House, 2001.

[16] A. Poggio and E. Miller, Computer Techniques for Electromagnetics. Pergamon Press, 1973.

[17] R. Fernandez-Recio, D. Escot-Bocanegra, D. Poyatos-Martinez, and M. Mulero-Valenzuela, "Technical report on the verification of radar cross section prediction of metallic targets by EADS Construcciones Aeronaticas S.A.” ED/RPT/4230/017/INTA/11, 2011.

[18] S. G. Garcia, J. Alvarez, L. D. Angulo, and M. R. Cabello, "UGRFDTD EM solver," 2011. [Online]. Available: http://maxwell.ugr.es/ugrfdtd/

[19] H. S. Consortium, "HIRF SE project," 2008. [Online]. Available: http://www.hirf-se.eu

[20] Workshop EM JINA, Radar Signatures, Nice, France, November 2006.

[21] F. L. Teixeira and W. C. Chew, "Analytical derivation of a conformal perfectly matched absorber for electromagnetic waves," Microwave Optical Technology Letters, vol. 17, no. 4, pp. 231-236, 1998.

[22] O. Ergul and L. Gurel, "Rigorous Solutions of Electromagnetic Problems Involving Hundreds of Millions of Unknowns," IEEE Antennas and Propagation Magazine, vol. 53, pp. 18-27, 2011. 